\title{
Quantificação de macro e micro nutrientes em algumas etnovariedades de cubiu (Solanum sessiliflorum Dunal)
}

\author{
Lúcia K. O. YUYAMA', Sonja H. M. MACEDO, Jaime P. L. AGUIAR'; Danilo S. FILHO², \\ Kaoru YUYAMA², Déborah I. T. FÁVARO³, Marina B. A. VASCONCELLOS 3 .
}

\begin{abstract}
RESUMO
Considerando a ampla variabilidade genética de cubiu (Solanum sessiliflorum Dunal), quantificaram-se os macro e micronutrientes, objetivando a ampliação da tabela de composição química de alimentos típicos da região amazônica. Os frutos provenientes da Estação Experimental de Hortaliças Alejo von der Pahlen (EEH) do Instituto Nacional de Pesquisas da Amazônia (INPA), localizados no km 14 da Rodovia AM 010 em Manaus, AM, foram processados no Laboratório de Alimentos e Nutrição do INPA. Avaliaram-se oito etnovariedades de cubiu identificados como: 2 I, 3 I, 6, 7, 12, 14, 17, 29 I e III em estádio de maturação comercial. Os teores de elementos minerais foram quantificados pela técnica de Ativação por Nêutrons Instrumental e a fibra alimentar pelo método enzímico-gravimétrico. Os resultados demonstram ser o cubiu um fruto com baixo conteúdo energético (média de $33 \mathrm{kcal}$ ), com conteúdo de fibra alimentar total na ordem de 1,6\%. Em relação aos macros elementos minerais, a etnovariedade 6 , apresentou a maior concentração em potássio $(513,5 \pm 3,1 \mathrm{mg})$, cálcio $(18,9 \pm 0,6 \mathrm{mg})$ e a etnovariedade $2 \mathrm{I}$ em Fe $(564,4 \pm 58,1 \mu \mathrm{g})$ e $\mathrm{Cr}(99,3 \pm 8,3 \mu \mathrm{g})$. A menor concentração foi constatada na etnovariedade 12 para os elementos $\mathrm{K}(229,0 \pm 4,5 \mathrm{mg}), \mathrm{Na}(53,7 \pm 5,5 \mu \mathrm{g})$ e $\mathrm{Zn}(89,3 \pm 4,7 \mu \mathrm{g})$. Apesar das variações em relação as diferentes etno variedades e conseqüentemente concentrações em elementos minerais, o cubiu, pode estar contribuindo para atingir as recomendações desses nutrientes.
\end{abstract}

PALAVRAS-CHAVE

Solanum sessiliflorum, Minerais, Composição centesimal, Análise por ativação com nêutrons.

\section{Macro and micro nutrients quantification of some cubiu ethnovarieties (Solanum sessiliflorum Dunal)}

\begin{abstract}
Considering the wide genetic variability of cubiu (Solanum sessiliflorum Dunal), its chemical composition was quantified in order to contribute to the chemical composition table of typical Amazonian foods. The cubiu fruit was collected at Alejo von der Pahlen (EEH) experimental station from National Research Institute of Amazonia (INPA). Eight ethno varieties of cubiu identified as 2 I, 3 I, 6, 7, 12, 14, 17, 29 I and III were analyzed. All samples used were ripe. The mineral content was determined by Neutron Activation Analysis technique and alimentary fiber by the enzymatic-gravimetric method. The results showed that cubiu is a hypo caloric food source (mean of $33 \mathrm{kcal}$ and total fiber of about $1.6 \%$ ). Regarding macronutrients, ethnovariety 6 showed high potassium $(513.5 \pm 3.1 \mathrm{mg})$ and calcium $(18.8 \pm 0.6 \mathrm{mg})$ concentration. Ethnovariety 21 , had high $\mathrm{Fe}(564 \pm 58 \mu \mathrm{g})$ and $\mathrm{Cr}(99 \pm 8 \mu \mathrm{g})$ concentrations. Low concentrations of $\mathrm{K}(229.0 \pm 4.5 \mathrm{mg}), \mathrm{Na}(53.7 \pm 5.5 \mu \mathrm{g})$ and $\mathrm{Zn}(89.3 \pm 4.7 \mu \mathrm{g})$ were found for ethnovariety 12 . Although the cubiu fruit presents great variation of its mineral contents due to different ethnovarieties, it could still contribute as a diet supplement to reach the minimum nutrient requirements for the Amazonian population.
\end{abstract}

KEYWORDS

Solanum sessiliflorum, Mineral content, Proximate composition, Neutron activation analysis technique.

\footnotetext{
1 Instituto Nacional de Pesquisas da Amazônia - INPA/CPCS. Av. André Araújo 2936. Petrópolis, Manaus-AM. CEP- 69.083-000. e-mail: yuyama@inpa.gov.br

2 Instituto Nacional de Pesquisas da Amazônia - INPA/CPCA. Av. André Araújo 2936. Petrópolis, Manaus-AM. CEP- 69.083-000

${ }^{3}$ Comissão Nacional de Energia Nuclear- CNEN/IPEN-SP. CP/. 11049. CEP- 05422-970, São Paulo.
} 


\section{INTRODUÇÃO}

A Região Amazônica se apresenta como a maior detentora em recursos naturais com inúmeras fruteiras com grande potencial econômico e nutritivo (Aguiar et al., 1980; Aguiar, 1996; Yuyama et al., 1997). Contudo, há muito que se estudar particularmente em relação ao cubiu (Solanum sessiliflorum Dunal). O cubiu é uma planta nativa da Amazônia Ocidental, tipo herbácea, presente em toda a Amazônia Brasileira, Peruana e Colombiana. As inúmeras etnovariedades originando frutos de diferentes formatos e tamanhos induzem a busca de conhecimentos que caracterizem as variações morfológicas e a composição química, possibilitando melhor conhecimento da biologia da espécie e da constituição genética de suas etnovariedades de forma a atender a demanda e a exigência da agroindústria (Silva Filho et al., 1997). O cubiu dependendo do genótipo pode atingir até 100 toneladas por hectare, com a vantagem de ser facilmente cultivada. De sabor e aroma agradáveis, os frutos são consumidos in natura como tira gosto, na forma de saladas e sucos, doces, geléias, compotas e tempero de peixe e carnes (Silva Filho et al., 1997).

Considerando a limitação de informaçōes sobre o valor nutricional das diferentes etnovariedades, salienta-se a necessidade da quantificação desses constituintes químicos, elementos importantes não só no processo da agroindústria, mas no resgate cultural de uma espécie autóctone. Dessa forma, o presente estudo quantificou os constituintes nutricionais do cubiu objetivando contribuir na disponibilização de uma Tabela de Composição Química de Alimentos para a região Amazônica e ao Brasil.

\section{MATERIAL E MÉTODOS}

Os frutos foram provenientes da Estação Experimental de Hortaliças Alejo von der Pahlen (EEH) do Instituto Nacional de Pesquisas da Amazônia (INPA), localizada no km 14 da Rodovia AM 010 em Manaus, AM. Foram avaliadas oito etnovariedades de Cubiu (S.sessiliflorum Dunal) identificados como: 2 I, 3 I, 6, 7, 12, 14, 17, 29 I e III em estádio de maturação comercial. O solo dessa área é do tipo Podzólico Vermelho-Amarelo álico, textura arenosa, de baixa fertilidade e o clima local caracterizado como "Afi" no esquema de Köppen, registrando $2.450 \mathrm{~mm}$ de chuva, com uma estação seca no período de julho a setembro (EMBRAPA, 1982).

Os frutos destinados às avaliações químicas e físicoquímicas, foram processados no Laboratório de Alimentos e Nutrição da Coordenação de Pesquisas em Ciências da Saúde - CPCS do Instituto Nacional de Pesquisas da Amazônia - INPA. O processamento consistiu na lavagem dos frutos em água corrente, retirada dos pedúnculos, casca e placenta por meio da utilização de faca de aço inoxidável, seguida da secagem em estufa com circulação de ar forçada a $60^{\circ} \mathrm{C}$, para fins de determinação do teor de umidade. A fim de minimizar a contaminação por metais, os utensílios, bandejas de aço inoxidável e vidrarias foram desmineralizados com EDTA (Ácido Etilenodiaminotetracético) a $1 \%$ e ácido nítrico a $30 \%$ respectivamente, seguido do enxágüe por no mínimo seis vezes com água desionizada e secagem em estufa com temperatura controlada e foram devidamente acondicionados até seu uso.

As características físicas de um total de 15 frutos selecionados aleatoriamente de um pool de diferentes etnovariedades foram analisadas segundo o diâmetro e altura tendo como instrumento de medida a fita métrica e o peso dos frutos em balança elétrica com capacidade de até cinco quilogramas. Em seguida, os resultados foram expressos em valores percentuais.

As determinações de umidade, cinza, lipídios e proteínas foram realizadas em triplicata de acordo com a metodologia preconizada pela AOAC (1995). A fração fibra alimentar foi quantificada de acordo com o método enzímico-gravimétrico de ASP et al (1983) e analisados separadamente os teores de fibra alimentar solúvel e insolúvel.

Os percentuais de carboidratos foram calculados por diferença dos outros componentes centesimais.

Uma alíquota das amostras de cubiu foi enviada ao Laboratório de Ativação Neutrônica do Instituto de Pesquisas Energéticas e Nucleares - IPEN/ CNEN, São Paulo, e os elementos minerais quantificados pela técnica de Análise de Ativação com Nêutrons Instrumental (AANI), conforme descrição no trabalho de Favaro et al (2000). A validação da metodologia de (AANI) foi feita por meio da análise do material de referência certificado OYSTER TISSUE (OT) (NIST SRM 1566). Para fins de estatística foi utilizada a análise de variância pelo teste $\mathrm{F}$ e o teste de Tukey com 5\% de probabilidade, para fins de comparação entre as médias das diferentes etnovariedades de cubiu (Pimentel Gomes, 1987).

\section{RESULTADOS E DISCUSSÃO}

Com relação aos dados de composição centesimal, as etnovariedades de cubiu analisadas apresentaram altos teores de umidade, na ordem de $90 \%$ com variação de 88,4 a $92,1 \%$, baixa concentração de proteínas $(0,4$ a $0,7 \%)$ e lipídios $(0,3$ a $1,8 \%$ ) (Tabela 1). Esses resultados evidenciam que o material genético pesquisado independente da origem e solo, várzea ou terra firme, mantém uma das características marcantes do fruto que é o alto teor de umidade, corroborando com os estudos de Silva Filho (1994) e Silva Filho et al. (1997 e 1989) cuja variação foi de 86 a $93 \%$.

Do ponto de vista industrial, a viabilidade econômica vai depender de etnovariedades que apresentem menor concentração de umidade. Contudo, não foram identificadas 
Tabela 1 - Composição centesimal da polpa, de 8 etnovariedades de cubiu (S. Sessiliflorum Dunal) em $100 \mathrm{~g}$ de polpa.(1)

\begin{tabular}{|c|c|c|c|c|c|c|c|c|c|}
\hline Etn. & Umidade & Cinza & Proteína & Extrato Etéreo & Fibra Insolúvel & Fibra Solúvel & Fibra Total & $\begin{array}{l}\text { Carboidra } \\
\text { tos(2) }\end{array}$ & $\begin{array}{l}\text { Energia } \\
\text { (kcal) }\end{array}$ \\
\hline 21 & 91,7 & 0,6 & 0,4 & 0,6 & 1,3 & 0,9 & 2,2 & 4,5 & 25,0 \\
\hline 31 & 90,7 & 0,5 & 0,4 & 0,8 & 1,3 & 0,07 & 1,4 & 6,2 & 33,6 \\
\hline 6 & 88,4 & 0,7 & 0,7 & 1,3 & 1,8 & 0,05 & 1,8 & 7,1 & 42,9 \\
\hline 7 & 90,5 & 0,7 & 0,5 & 1,1 & 1,4 & 0,1 & 1,5 & 5,7 & 34,7 \\
\hline 12 & 92,1 & 0,5 & 0,4 & 0,3 & 1,3 & 0,3 & 1,6 & 5,1 & 24,7 \\
\hline 14 III & 91,0 & 0,5 & 0,4 & 0,5 & 1,4 & 0,2 & 1,6 & 6,0 & 29,1 \\
\hline 17 & 89,5 & 0,7 & 0,7 & 0,9 & 1,6 & 0,5 & 2,1 & 6,1 & 35,3 \\
\hline 291 & 89,8 & 0,6 & 0,6 & 1,8 & 0,9 & 0 & 0,9 & 6,3 & 43,8 \\
\hline \multirow[t]{2}{*}{ Média } & 90,5 & 0,6 & 0,5 & 0,9 & 1,3 & 0,3 & 1,6 & 5,9 & 33,7 \\
\hline & 1,3 & $\pm 0,09$ & $\pm 0,1$ & $\pm 0,4$ & $\pm 0,2$ & $\pm 0,3$ & $\pm 0,4$ & $\pm 0,8$ & $\pm 7,1$ \\
\hline
\end{tabular}

1: com base em $100 \mathrm{~g}$ de material fresco. 2: calculado por diferença dos demais componentes. $\pm=$ Desvio Padrão (DP). Etn $=$ Etnovariedades

etnovariedades que se destacassem nesta característica específica, a não ser as populaçôes 6 e 25 como as mais promissoras, o que significa um ganho de $18 \mathrm{~kg}$ de polpa/tonelada. Por outro lado, sob o ponto de vista nutricional, considerando as preocupações com as doenças crônicas nutricionais, como obesidade, diabetes, doenças cardiovasculares e certos tipos de cânceres, o fruto pode ser mais uma opção para compor o cardápio do amazonense com restrição energética.

A baixa concentração de proteínas e lipídios (Tabela 1) nos frutos analisados deixa implícita a pouca contribuição das solanáceas neste constituinte nutricional, conforme constatado em amostras de outras regióes (Pahlen, 1977; Carbajal, 1988).

O conteúdo de fibra alimentar total no cubiu apresentou-se em maior proporção, apesar da variação entre os teores de fibra solúvel e insolúvel para as etnovariedades analisadas (Tabela 1). Os valores médios, na ordem de 1,6\% desses constituintes, se aproximam aos das espécies do mesmo gênero como a beringela ( $S$. melongena L.) com 1,2\% na polpa integral e jiló (S. gilo Raddi) 1,2 \% (ENDEF, 1981), evidenciando assim, a necessidade de estudos mais aprofundados em relação às funções fisiológicas do cubiu como fonte de fibra.

A baixa concentração de carboidratos e conseqüentemente o baixo valor energético do cubiu (Tabela 1) são características típicas do fruto e não diferem dos reportados na literatura (Pahlen, 1977, Villachica,1996). Tais achados podem compor um banco de dados de frutos amazônicos com baixa densidade energética, constituindo assim, em opçôes para nortear a alimentação de indivíduos com sobrepeso e obesidade ou aqueles com restrição energética.

Entre os macros elementos minerais analisados (Tabela 2), evidencia-se maior concentração de potássio com variação de 229,0 a $513,5 \mathrm{mg} \%(\mathrm{p}<0,05)$. Os valores encontrados para o potássio corroboram com os estudos de Yuyama et al. (1997), envolvendo outras espécies de consumo popular como manga, graviola e cupuaçu. Considerando a recomendação de 4700 mg.d $\mathrm{d}^{-1}$, de potássio para um homem adulto entre $31-50$ anos, $100 \mathrm{~g}$ de polpa de cubiu representa $5-11 \%$ desta necessidade, de acordo com as recomendaçôes do (NAS/FNB/IOM, 2003). As variaçôes nas concentrações desses elementos permitem a seleção de etnovariedades mais apropriadas para seu emprego em dietas hiper ou hipo calêmicas. Dentre os macroelementos analisados, sódio foi o que apresentou a menor concentração dentre as etnovariedades analisadas, variando de 53,7 a 336,4 $\mu \mathrm{g} \%$ (Tabela 2). Tais constataçóes corroboram com os valores citados por Villachica (1996). Se a recomendação é de $1500 \mathrm{mg}$ para um homem adulto no estágio de vida de 31-50 anos (NAS/FNB/IOM, 2003), o cubiu pouco contribui como alimento fonte de sódio. No entanto, é uma opção importante aos pacientes com restrição desse elemento.

A concentração de cálcio presente no cubiu, é similar aos citados por Pahlen (1977) e Villachica (1996) e a de outros frutos populares como a manga e o cupuaçu (Yuyama et al, 1997). A variação na concentração de cálcio, na ordem de 8,51 a $18,8 \mathrm{mg} \%$, permite a seleção de variedades mais apropriadas para produção industrial, por se tratar de um elemento que participa ativamente na complexação da pectina durante a formação do gel, particularmente quando se deseja formular e processar produtos com baixo teor de açúcar, como os dietéticos (Jackix, 1988).

Dentre os micronutrientes analisados, mesmo o ferro sendo o elemento em maior concentração no cubiu, com uma média de 423,5 $\mu \mathrm{g} \%$ (Tabela 2), esses valores são inexpressivos e inferiores aos encontrados por Pahlen (1977) e Villachica (1996). Obviamente vários são os fatores que interferem na variação desse elemento como a etnovariedade, tipo de solo, condições edafoclimáticas, tratos culturais e metodologias. Sendo a recomendação diária de $8 \mathrm{mg}$. dia $^{-1}$ de ferro, para um homem adulto (NAS/FNB/ IOM, 2001), o cubiu representa cerca de $5 \%$ da ingestão diária.

Em relação ao zinco, a variação de concentração nas amostras analisadas (Tabela 2), demonstra certa semelhança com os demais frutos amazônicos de acordo com os estudos 


\begin{tabular}{|c|c|c|c|c|c|c|c|}
\hline Etn & $\begin{array}{l}\text { Fe }(\mu \mathrm{g}) \\
X \pm D P\end{array}$ & $\begin{array}{l}Z n(\mu g) \\
X \pm D P\end{array}$ & $\begin{array}{l}\text { Co }(\mu \mathrm{g}) \\
X \pm D P\end{array}$ & $\begin{array}{l}\mathrm{Cr}(\mu \mathrm{g}) \\
\mathrm{X} \pm \mathrm{DP}\end{array}$ & $\begin{array}{l}\mathrm{Ca}(\mathrm{mg}) \\
\mathrm{X} \pm \mathrm{DP}\end{array}$ & $\begin{array}{l}\mathrm{K}(\mathrm{mg}) \\
\mathrm{X} \pm \mathrm{DP}\end{array}$ & $\begin{array}{l}\mathrm{Na}(\mu \mathrm{g}) \\
X \pm D P\end{array}$ \\
\hline 7 & $218,5 \pm 19,0^{\mathrm{e}}$ & $115,9 \pm 7,6^{\text {cd }}$ & $427,5 \pm 28,5^{d}$ & $18,0 \pm 0,9^{c}$ & $15,9 \pm 2,5^{a}$ & $362,6 \pm 47,6^{a}$ & $123,5 \pm 9,5^{c}$ \\
\hline 17 & $346,5 \pm 10,5^{d}$ & $144,9 \pm 6,3^{a}$ & $661,5 \pm 84,0^{c}$ & $33,6 \pm 4,2^{c}$ & $8,5 \pm 0,7^{c}$ & $380,4 \pm 17,5^{\mathrm{ab}}$ & $116,6 \pm 4,2^{\text {cd }}$ \\
\hline 291 & $489,6 \pm 51,0^{c}$ & $131,6 \pm 8,2^{a b}$ & $867,0 \pm 81,6^{b}$ & $73,4 \pm 7,1^{b}$ & $10,9 \pm 13,1^{b c}$ & $380,3 \pm 49,0^{a}$ & $80,6 \pm 3,1^{\mathrm{de}}$ \\
\hline 14 III & $477,0 \pm 18,0^{\text {bc }}$ & $126,0 \pm 5,4^{b c}$ & $792,0 \pm 45,0^{a b}$ & $78,3 \pm 13,5^{b}$ & $13,1 \pm 0,5^{\mathrm{a}}$ & $341,1 \pm 2,3^{a}$ & $83,7 \pm 8,1^{\text {de }}$ \\
\hline 31 & $530,1 \pm 27,9^{b}$ & $101,4 \pm 2,8^{d}$ & $725,0 \pm 55,8^{b}$ & $70,7 \pm 7,4^{b}$ & $9,8 \pm 0,4^{c}$ & $343,6 \pm 22,3^{a b}$ & $156,2 \pm 8,4^{b}$ \\
\hline 12 & $379,2 \pm 15,8^{c}$ & $89,3 \pm 4,7^{d}$ & $608,3 \pm 47,4^{b}$ & $63,2 \pm 3,2^{b}$ & $11,0 \pm 1,9 a b$ & $229,0 \pm 4,5^{b}$ & $53,7 \pm 5,5^{\mathrm{e}}$ \\
\hline 21 & $564,4 \pm 58,1^{a}$ & $127,8^{ \pm} \pm 7,5^{b}$ & $830,0 \pm 74,7^{a}$ & $99,6 \pm 8,3^{a}$ & $12,3 \pm 1,3^{a}$ & $300,7 \pm 24,8$ ab & $74,7 \pm 8,3^{\mathrm{de}}$ \\
\hline 6 & $382,8 \pm 11,6^{d}$ & $140,4 \pm 3,5^{\mathrm{ab}}$ & $545,2 \pm 34,8^{d}$ & $33,6 \pm 2,3^{c}$ & $18,8 \pm 0,6^{a}$ & $513,5 \pm 3,1^{\mathrm{a}}$ & $336,4 \pm 23,2^{a}$ \\
\hline Média & $423,51 \pm 113,40$ & $122,16 \pm 19,03$ & $682,06 \pm 150,91$ & $65,47 \pm 33,05$ & $12,54 \pm 3,37$ & $356,40 \pm 80,71$ & $128,17 \pm 90,20$ \\
\hline
\end{tabular}

Letras iguais seguidas no sentido vertical não diferem entre si em nível de $5 \%$ de significância pelo teste de Tukey. $\pm=$ Desvio Padrão (DP) Etn $=$ Etnovariedades

Tabela 3 - Teores de Microminerais em algumas etnovariedades de cubiu (S. sessiliflorum) em $100 \mathrm{~g}$ de polpa integral.

\begin{tabular}{llll}
\hline Etn & $\begin{array}{l}\mathrm{Rb}(\mu \mathrm{g} / 100 \mathrm{~g}) \\
\mathrm{X} \pm \mathrm{DP}\end{array}$ & $\begin{array}{l}\mathrm{Ba}(\mu \mathrm{g} / 100 \mathrm{~g}) \\
\mathrm{X} \pm \mathrm{DP}\end{array}$ & $\begin{array}{l}\mathrm{Br}(\mu \mathrm{g} / 100 \mathrm{~g}) \\
\mathrm{X} \pm \mathrm{DP}\end{array}$ \\
\hline 7 & $969,0 \pm 76,0^{\mathrm{abc}}$ & $92,1 \pm 7,6^{\mathrm{a}}$ & $47,5 \pm 1,9^{\mathrm{b}}$ \\
\hline 17 & $1165,5 \pm 84,0^{\mathrm{a}}$ & $189,0 \pm 31,5^{\mathrm{a}}$ & $51,4 \pm 2,1^{\mathrm{bc}}$ \\
29 & $1152,6 \pm 91,8^{\mathrm{a}}$ & $98,9^{\mathrm{a}} \pm 6,1^{\mathrm{a}}$ & $26,5 \pm 0,6^{\mathrm{e}}$ \\
\hline $14 \mathrm{III}$ & $828,0 \pm 900,0^{\mathrm{bc}}$ & $126,0 \pm 27,0^{\mathrm{a}}$ & $55,8 \pm 2,7^{\mathrm{a}}$ \\
$3 \mathrm{I}$ & $1013,0 \pm 46,5^{\mathrm{ab}}$ & $120,9 \pm 9,3^{\mathrm{a}}$ & $35,3 \pm 0,9^{\mathrm{d}}$ \\
12 & $734,7 \pm 47,4^{\mathrm{bc}}$ & $120,9 \pm 1,6^{\mathrm{a}}$ & $34,0 \pm 5,5^{\mathrm{cd}}$ \\
$2 \mathrm{I}$ & $747,0 \pm 33,2^{\mathrm{c}}$ & $106,2 \pm 7,5^{\mathrm{a}}$ & $23,5 \pm 0,7^{\mathrm{e}}$ \\
\hline 6 & $1310,8 \pm 69,6^{\mathrm{a}}$ & $139,2 \pm 34,8^{\mathrm{a}}$ & $47,0 \pm 0,6^{\mathrm{d}}$ \\
\hline Média & $990,07 \pm 210,98$ & $124,15 \pm 30,34$ & $40,12 \pm 11,94$ \\
\hline
\end{tabular}

Letras iguais no sentido vertical não diferem entre si em nível de $5 \%$ de significância pelo teste de Tukey.

Etn= Etnovariedades

de Yuyama et al.(1997), ou seja, os referidos frutos são fontes limitadas desse elemento mineral.

Os elementos rubídio, bário e bromo (Tabela 3) ficam como contribuição para efeito de comparação em estudos futuros, pois não apresentam valores de recomendação.

Nas condições do presente estudo e para as espécies analisadas os valores encontrados, mesmo que em menor quantidade, podem estar somando na alimentação dos amazonenses.

\section{CONCLUSÕES}

Dentre as etnovariedades estudadas, a de número 6 , seguida da 2 I foram as que apresentaram as maiores concentrações de elementos minerais (macro e micro), sendo portanto, um recurso genético para a agroindústria e para programas que visem a curto prazo, a melhoria da alimentação da população da região Amazônica, em particular os grupos carentes nesses elementos minerais.
A quantidade de fibra alimentar presente no cubiu e a baixa densidade energética sugere a utilização do mesmo em dietas hipocalóricas.

\section{AGRADECIMENTOS}

Ao INPA pelo apoio logístico, ao CNPq pela concessão da bolsa (Proc. 302811/2003-6) e pelo apoio financeiro (Proc. 476293/2003-0).

\section{BIBLIOGRAFIA CITADA}

Aguiar, J.P.L.; Marinho, H.A.; Rebelo, Y.S.; Shrimpton, R. 1980. Aspectos nutritivos de alguns frutos da Amazônia. Acta Amazonica, 10(4): 755-758.

Aguiar, J.P.L. 1996. Tabela de composição de Alimentos da Amazônia. Acta Amazonica, 26(1/2): 121-126.

Asp, N.G.; Johansson, C.G.; Hallmer, H; Siljestrom, M. 1983. Rapid enzymatic assay of insoluble and soluble dietary fiber. J. Agric. Food. Chem., 31(3): 476-482.

AOAC - ASSOCIATION OF OFFICIAL ANALYTICAL CHEMISTS. 1995. Oficial methods of analysis. 16.ed., Arlington. 937pp.

Carbajal, W.F. Estudo técnico para la elaboración de pulpa, néctar y jalea a partir de la cocona (Solanum sessiliflorum Dunal). 1988. Monografia, Facultad de Ingeniería em Industrias Alimentarias, Universidad Nacional de la Amazonía Peruana, Iquitos. 94pp.

EMBRAPA, 1982. Boletim agrometeorológico. Manaus, EMBRAPA/ UEPAE. 22pp.

ENDEF - ESTUDO NACIONAL DA DESPESA FAMILIAR. Tabelas de Composição de Alimentos. 1981. 2.ed. Fundação Instituto Brasileiro de Geografia e Estatística - IBGE. Rio de Janeiro/RJ. 213pp.

Favaro, D.I.T.; Maihara, V.A.; Mafra, D.; Souza, S.A.; Vasconcellos, M.B.A.; Cordeiro, M.B.C.; Cozzolino, S.M.F. 2000. Application of NAA to the determination of mineral and trace elements in Brazilian diets at IPEN/CNEN-SP. J. Radioanalytical and Nuclear Chemistry, 244(1): 241-245. 
Jackix, M.H. 1988. Doces, geléias e frutas em calda. São Paulo: Ícone. 172pp.

Nacional Academy of Science, Food and Nutrition Board, Institute of Medicine-NAS/FNB/ IOM 2003. dietary referente intakesdirs for water, potassium, sodio, chloride, and sulfate, copyright. http://www.nap.edu.

Nacional Academy of Science, Food and Nutrition Board, Institute of Medicine-NAS/FNB/ IOM. 2001. Dietary Reference Intakes for Vitamin A, Vitamin K, Arsenic, Boron, Chromium, Copper, Iodine, Iron, Manganese, Molybdenum, Nickel, Silver, Vanadium, and Zinc, copyrigh. http://www.nap.edu.

Pahlen, A.V.D. 1977. Cubiu [Solanum topiro (Humb \& Bonpl.)], uma fruteira da Amazônia. Acta Amazonica, 7(3): 301-307.

Pimentel Gomez, F. 1987. Curso de Estatística Experimental. 12 ed. Piracicaba (SP). 466pp.

Silva Filho, D.F. Variabilidade genética em 29 populaçôes de cubiu (Solanum topiro Humbl. \& Bonpl. Solanaceae) avaliada na zona da mata do Estado de Pernambuco. 1994. Dissertação de Mestrado, Universidade Federal Rural de Pernambuco, Recife, PE. 80pp.
Silva Filho, D.F.; Noda, H.; Paiva, W.O.; Yuyama, K.; Bueno, C.R.; Machado, F.M. 1997. Hortaliças não convencionais nativas e introduzidas na Amazônia. In: Noda, H.; Souza, L.A.G.; Fonseca, O.J.M. Duas décadas de contribuiçôes do INPA à pesquisa agronômica no trópico úmido. Manaus, AM. p.19-87.

Silva Filho, D.F.; Clement, C.R.; Noda, H. 1989. Variação fenotípica em frutos de doze introduções de cubiu (Solanum sessiliflorum Dunal) avaliadas em Manaus, AM. Brasil. Acta Amazonica, 19(4): 9-18.

Villachica, H. 1996. Frutales y hortalizas promisorias de la Amazonia. Lima: Tratado de Cooperacion Amazonica-Secretaria ProTempore, 367pp.

Yuyama, L.K.O.; Aguiar, J.P.L.; Macedo, S.H.M.; Gioia, T.B.; Yuyama, K.; Fávaro, D.I.T.; Afonso, C.; Vasconcellos, S.M.F.; Cozzolino, S.M.F. Determinação dos teores de elementos minerais em alimentos convencionais e não convencionais da Região Amazônica pela técnica de análise por ativação com nêutrons instrumental. Acta Amazonica, 27(3): 183-196.

Recebido em 02/01/2007

Aceito em 20/06/2007 